\title{
Predicting local plasmon resonances and geometries using autoencoder networks in complex nanoparticle assemblies
}

Kevin Roccapriore ${ }^{1}$, Maxim Ziatdinov ${ }^{2}$, Shin Hum $\mathrm{Cho}^{3}$, Delia Milliron ${ }^{4}$, Jordan Hachtel $^{5}$ and Sergei Kalinin $^{6}$

${ }^{1}$ Oak Ridge National Laboratory, United States, ${ }^{2}$ Computational Sciences and Engineering Division, Oak Ridge National Laboratory, Oak Ridge, Tennessee, United States, ${ }^{3}$ Samsung Electronics, United States, ${ }^{4}$ University of Texas at Austin, United States, ${ }^{5}$ Center for Nanophase Materials Sciences, Oak Ridge National Laboratory, United States, ${ }^{6}$ Center for Nanophase Materials Sciences, Oak Ridge National Laboratory, Oak Ridge, Tennessee, United States

Developing nanostructures with desired optical properties is critical to the field of nanophotonics. To pave the way towards stochastic design of nanoplasmonic structures, we establish the correlative relationship between local nanoparticle geometries and their plasmonic responses. By using an encoder-decoder neural networks within the framework of PyTorch, we demonstrate the predictions of spectra from geometries in the im2spec network, as well as the predictions of geometry from spectral inputs in the spec2im network [1].

Equipped with a scanning electron transmission electron microscope (STEM), we acquire an electron energy loss (EEL) spectrum image and its associated acquired high angle annular dark field (HAADF) image of a self-assembled monolayer of fluorine and tin doped indium oxide nanocrystal arrays [2]. By tiling the HAADF and associating an EEL spectrum with each tile as seen in Fig. 1(a) and (b), we generate image-spectrum pairs that are used to teach the network the correlative relationship between each. Each network encodes the images (spectra) to a small number of latent variables in a bottleneck fashion, then decodes the latent variables into spectra (images). The reduced descriptions contained in the so-called latent space can yield a surprising insight into the generative mechanisms of complicated plasmonic responses in nanoparticle arrays [3].

Shown in Figure 2 is the predictive ability for the selected dataset. Note that the number of latent dimensions can be chosen during network training. Here, two separate networks containing two and ten dimensions are each trained separately, then their predictability is assessed. More features can be encoded into a higher number of dimensions, which yields a better performance of prediction, however the relationships are more difficult to understand and physical intuition may be lost. Minimizing the number of latent dimensions while maintaining a sufficient level of performance is therefore preferred. Figure 2 (a-c) shows the spectral predictions for selected geometries previously unseen by the neural network, for both two and ten latent dimensions. Similarly, Figure 2 (d-f) demonstrates the geometric predictions for selected unseen spectra.

These relationships learned by use of the autoencoder allow predictions of spectra from geometries and vice versa, and ultimately enhance the design capabilities for plasmonic systems, and may even permit a route to solving the inverse design challenge in nanophotonics. 
(a)

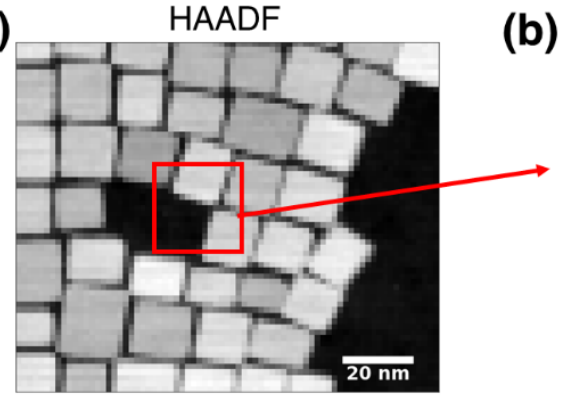

(b)

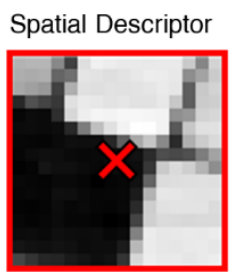

Spectral Descriptor

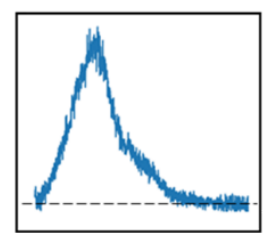

(c)

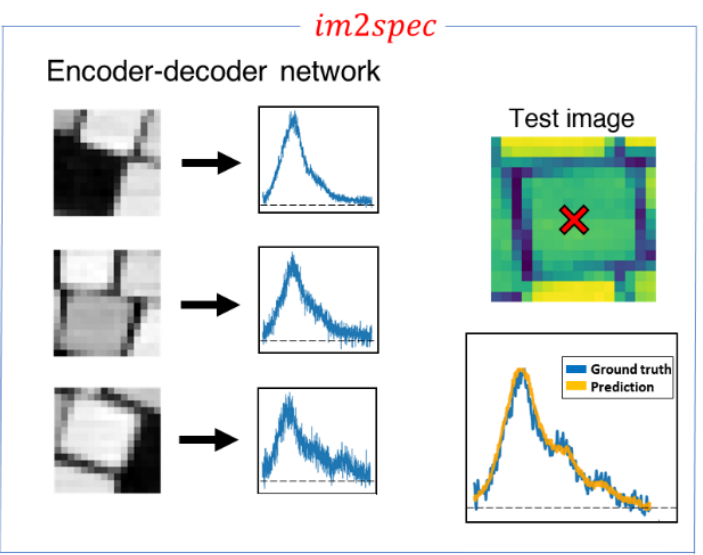

(d)

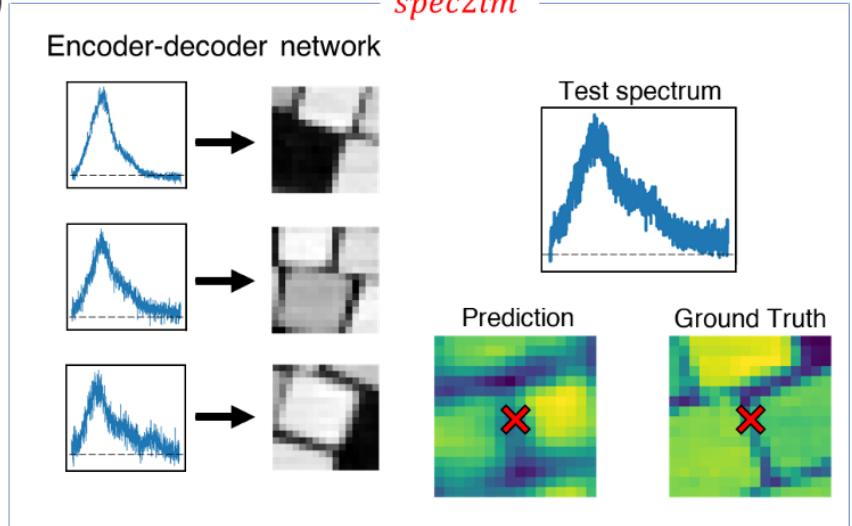

Figure 1. Figure 1. Principle of predicting plasmonic responses from local geometries and the inverse. (a) depicts a high angle annular dark field (HAADF) image captured simultaneously during electron energy loss spectrum image acquisition. HAADF is tiled into small sub-images, each with a corresponding reference spectrum, taken from the center pixel of the sub-image, shown in (b). Sub-images (spectra) are used as inputs into the im2spec (spec2im) autoencoder network, (c) and (d) respectively. The network encodes features into the so-called latent space, which is used to predict either spectra from local geometry (c) or local geometry from spectra (d). 
im 2 spec

Spectral prediction

(a)

(b)
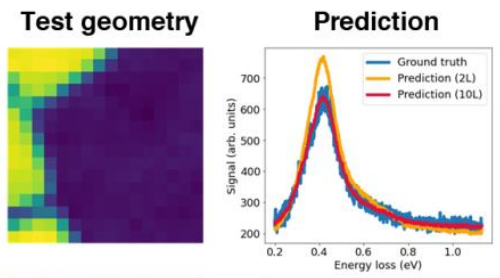

(c)
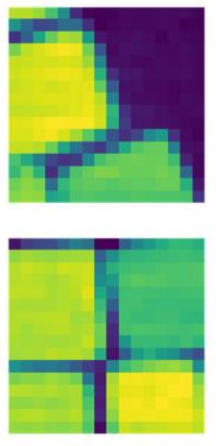
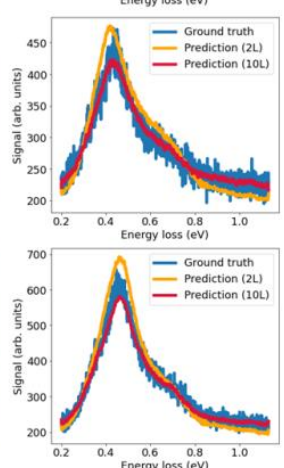

spec $2 \mathrm{im}$

Geometric prediction

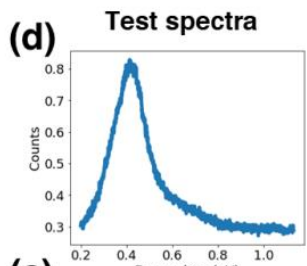

(e)
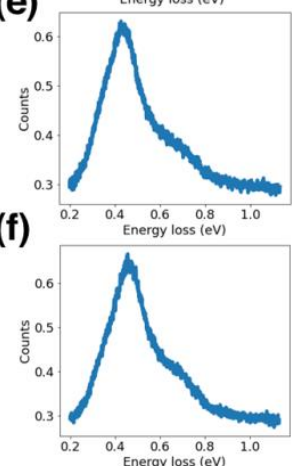
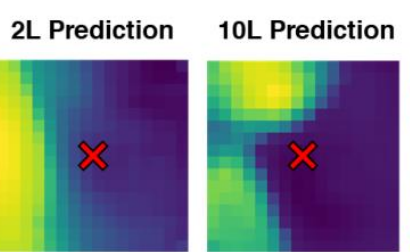

Ground Truth
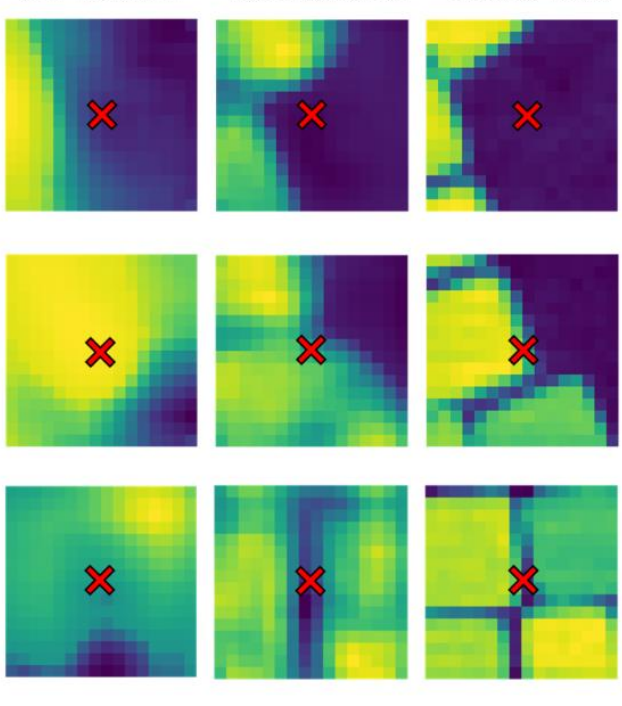

Figure 2. Figure 2. Spectral and geometric predictions of selected regions in Fig 1(a) using the im2spec and spec2im autoencoder architectures. (a-c) use local geometries the network has not previously encountered to predict the spectral output using either two or ten latent dimensions (separately trained). (d-f) use spectra the network has not previously encountered to predict the geometric arrangement that may give rise to such a response, again trained with two and ten latent variables. Ground truth spectra and geometry are shown in both panels.

\section{References:}

[1] S. V. Kalinin et al., arXiv2020 arXiv:2006.01374

[2] S. H. Cho et al., J., Chem. Mater.201931, 2661.

[3] K. M. Roccaprioreet al., arXiv2020 arXiv:2009.09005

[4] This effort (ML and STEM) is based upon work supported by the U.S. Department of Energy (DOE), Office of Science, Basic Energy Sciences (BES), Materials Sciences and Engineering Division (K.M.R., S.V.K.) and was performed and partially supported (J.A.H., M.Z.) at the Oak Ridge National Laboratory's Center for Nanophase Materials Sciences (CNMS), a U.S. Department of Energy, Office of Science User Facility. S.H.C acknowledges (NSF, CHE-19052631609656, CBET-1704634, NASCENT, an NSF ERC EEC-1160494, and CDCM, an NSF MRSEC DMR-1720595), the Welch Foundation (F-1848), and the Fulbright Program (IIE-15151071). 